\title{
TAROGE experiment and reconstruction technique for near-horizon impulsive radio signals induced by Ultra-high energy cosmic rays
}

\section{Yaocheng Chen* on behalf of the TAROGE Collaboration}

(a complete list of authors can be found at the end of the proceedings)

${ }^{a}$ Department of Physics and Leung Center for Cosmology and Particle Astrophysics, National Taiwan University, No. 1, Sec. 4, Roosevelt Rd., Taipei 10617, Taiwan, R.O.C.

E-mail: yaocheng.chencyc@gmail.com

\begin{abstract}
Taiwan Astroparticle Radiowave Observatory for Geo-synchrotron Emissions (TAROGE) is antenna arrays sitting on high coastal mountains of Taiwan, pointing to the Pacific Ocean for the detection of near-horizon extensive air showers (EAS) induced by ultra-high energy cosmic rays and Earth-skimming tau neutrinos. TAROGE would improve the detection capability by collecting both the direct emissions and the ocean-reflected signals on a vast area of ocean which is visible from Taiwan's high mountains. Four TAROGE stations in Taiwan have been deployed in the past few years. Angular resolution is crucial for discriminating between cosmic ray and neutrino events, the major systematic error of event direction reconstruction in TAROGE experiment comes from interference between direct and ground-reflected signals. We developed a new angular reconstruction method based on mapping and unfolding of differential response between channels which can be feasibly measured with a drone-borne calibration pulser. We achieved a sub-degree angular resolution for near horizon events. In this paper, we discuss details of the method and the results. A brief status report of the TAROGE project also will be reported.
\end{abstract}

$37^{\text {th }}$ International Cosmic Ray Conference (ICRC 2021)

July 12 th - 23rd, 2021

Online - Berlin, Germany

\footnotetext{
${ }^{*}$ Presenter
} 


\section{Introduction}

The study of Ultra-High Energy Cosmic Rays (UHECRs) with energies greater than $10^{18} \mathrm{eV}$ is an active research area where physicists hope to find the sources and the acceleration mechanisms of such high-energy particles. One effective way to observe UHECRs is by detecting radio pulses emitted from Extensive Air Shower (EAS) induced by them. The main mechanism of radio emission is geomagnetic effect. When charged particles of EAS propagating in the earth's magnetic field, the Lorentz force will deflect them and thus induces a transverse current. The time-varying transverse current will emit geomagnetic radiation which polarization is aligning with the Lorentz force[1]. This geomagnetic radiation is coherent at frequencies of a few to hundreds of $\mathrm{MHz}$, generating short transient pulses (a few nanoseconds) relativistically beamed in the EAS forward direction at a detectable level for showers with $10^{17} \mathrm{eV}$.

In addition to UHECRs, EAS can also be induced by the decay of tau leptons created through charged-current interaction of UHE (Ultra High Energy) neutrinos under the Earth's surface. UHE neutrinos could be produced through the interactions between cosmic rays and cosmic microwave background radiation (CMB), which is the so-called GZK effect named after Greisen, Zatsepin and Kuzmin. Unlike UHECRs will be deflected by intergalactic magnetic fields, the universe is almost transparent to UHE neutrinos, thus detecting UHE neutrinos is a good indirect strategy to discover UHECR sources. Many ground-based experiments have observed the suppression of the UHECR flux above $4 \cdot 10^{17} \mathrm{eV}[2][3]$, which supports the existence of the GZK effect.

Taiwan Astroparticle Radiowave Observatory for Geo-synchrotron Emissions (TAROGE) is a ground-based antenna array sitting on high coastal mountains of Taiwan, pointing to the Pacific Ocean for the detection of near-horizon extensive air showers (EAS) induced by UHECRs and Earth-skimming UHE $\tau$ neutrinos. Fig. 1 shows the detection concept of TAROGE. The unique cliff structure of TAROGE sites makes it capable of measuring not only the direct radio signal but also the signal reflected by the ocean [4]. Together with practical advantages such as low unit cost, easiness of deployment and maintenance, TAROGE presents appealing characteristics for the detection of near-horizon EAS. Four TAROGE stations in Taiwan have been deployed from 2014-2019, each station has improvements in instruments to increase detection efficiency. The latest deployed station TAROGE-4 has been equipped with a new trigger system by using Surface Acoustic Wave (SAW) filters based multi-bands coincidence technique. This new trigger system provides an effective discriminating power for impulsive geo-synchrotron signals against suburban anthropogenic backgrounds.

The major systematic error of event source direction reconstruction in TAROGE-4 comes from ground reflection effect. Conventional interferometric method based on waveform crosscorrelation[7] for near-horizon calibration events leads to a mis-reconstruction result, bias about $4^{\circ}$ in elevation angle. The event source direction is extremely important for discriminating upwardgoing and downward-going signals. One way to deal with this ground reflection effect is through deconvolution of its response which in principle can be modeled from ground surface geometry and soil electromagnetic properties. But in practice those parameters are onerous to obtain and the model-dependent ground reflection response will introduce another systematic uncertainty. We've developed a new algorithm based on mapping and folding of differential response between channels which can be feasibly and accurately measured through drone-borne calibration system. With this 


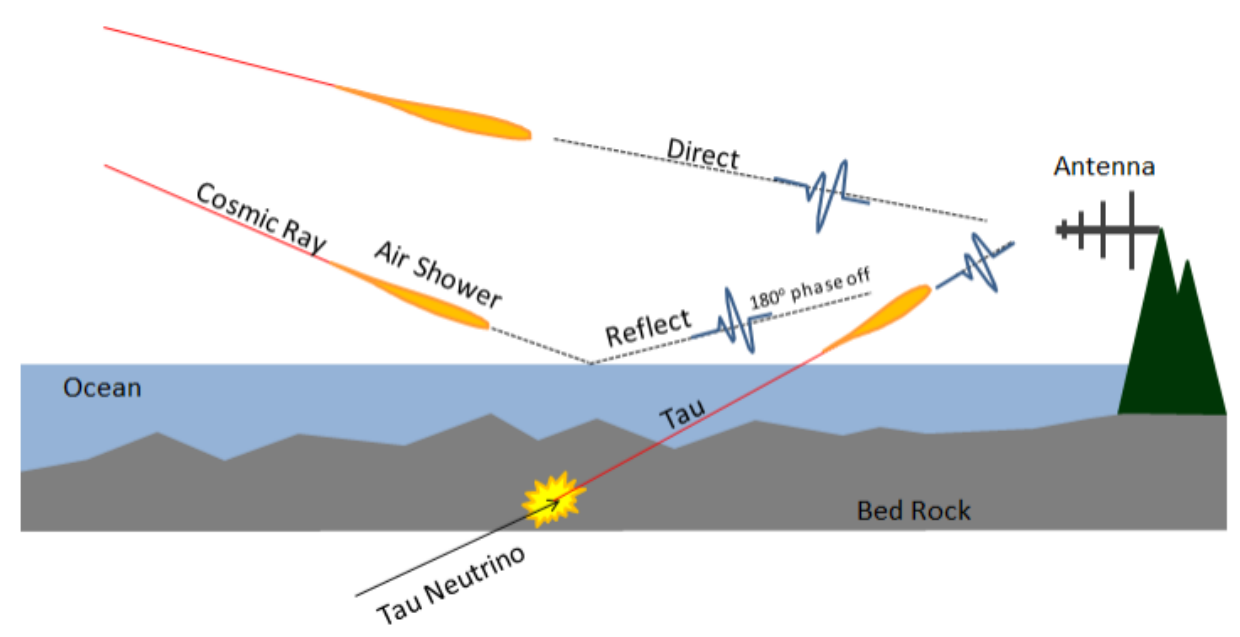

Figure 1: The detection concept of TAROGE, Adapted from [4].

novel method, TAROGE-4 has achieved sub-degree angular resolution for near-horizon events. Instrument of TAROGE-4 and detail angular reconstruction algorithm are presented in this paper.

\section{TAROGE-4 instrument}

TAROGE-4 station (as shown in Fig. 2) consists of 4 Christmas-tree-shape dual-polarized logperiodic dipole array (LPDA) antennas of bandwidth 180-350 MHz and $7 \mathrm{dBi}$ gain mounted on 3.5 $\mathrm{m}$ high towers. The whole system architecture is depicted in Fig. 3. Each antenna polarization has its own signal chain and the received signal is fed into Radio Frequency (RF) front-end electronics (FEE) which consists of a 180-350 MHz band-pass filter and a $63 \mathrm{~dB}$ gain low-noise-amplifier (LNA) and then processed by the data-acquisition (DAQ) system. Inside DAQ, the RF signals are split into two paths for trigger system and digitizers respectively. The digitizer system provides 8 channels with 1500 samples for each, 12-bits dynamic range, and with sampling rate set to 1.25 GHz. This autonomous station is powered by photovoltaic system and the communication with lab service is through $4 \mathrm{G}$ network.

The major improvement of TAROGE-4 is the trigger system, which uses SAW filters based multi-bands coincidence technique. Signal in each channel is divided into 8 sub-bands by SAW filters and each sub-band signal strength is measured by a power detector. The channel-level trigger is formed when at least 5 sub-band signal strength passes its threshold. This provides an effective discriminating power for impulsive signals against anthropogenic continue wave background since TAROGE-4 is nearby suburban area. Then the system trigger requires 3-out-of-4 coincidence of channel-level triggers within $60 \mathrm{~ns}$ for either H-pol or V-pol. The DAQ computer keeps monitoring and auto adjusting the threshold of each sub-band for targeting $10 \mathrm{~Hz}$ event rate.

\section{Event Reconstruction}

TAROGE-4 angular reconstruction performance is degraded by interference between direct and ground-reflected signals especially for near-horizon events which have about $4^{\circ}$ bias. Instead 


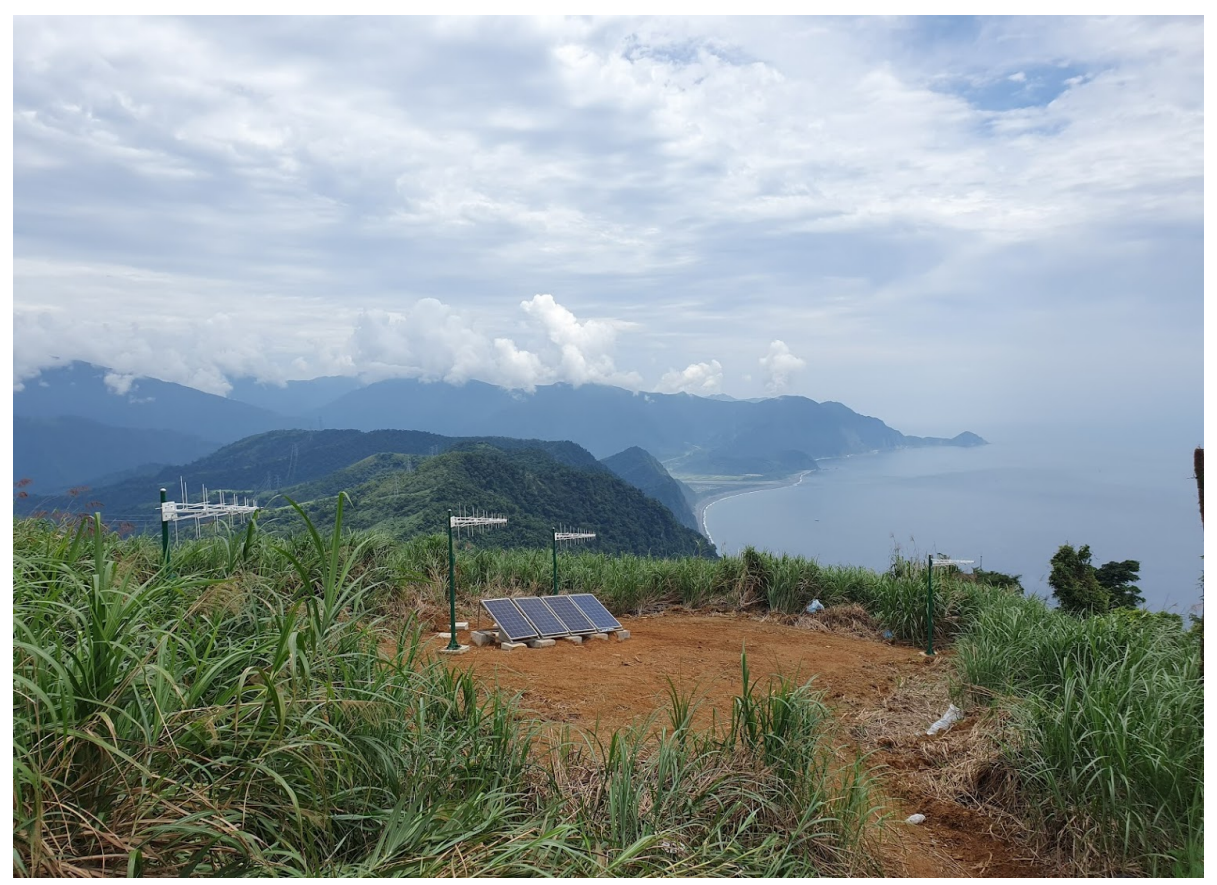

Figure 2: TAROGE-4 station, LPDA antennas pointing horizontally to ocean.

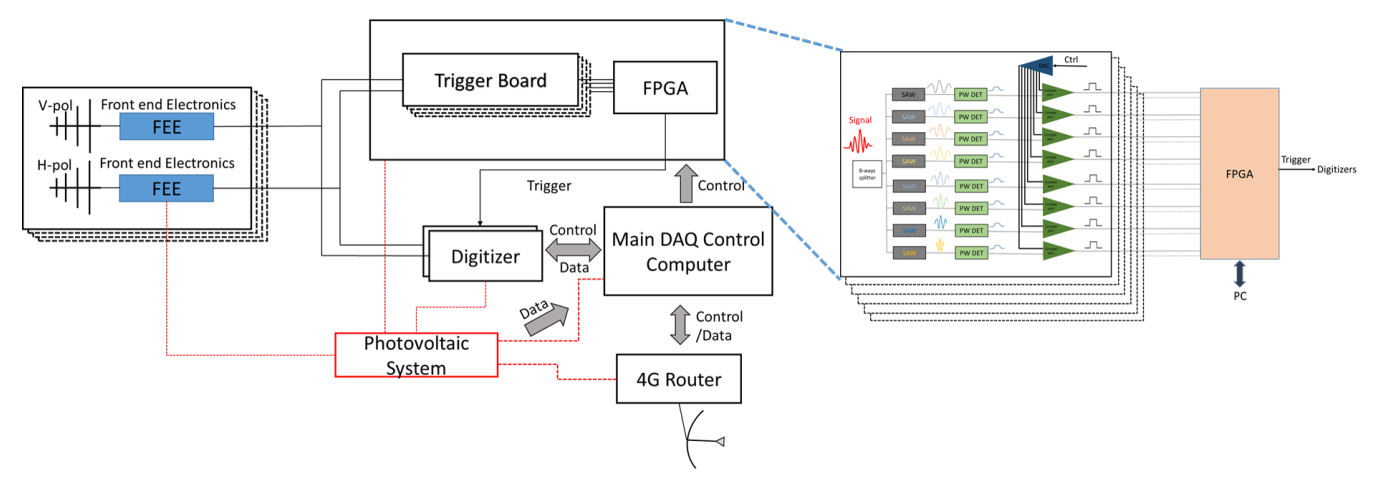

Figure 3: TAROGE-4 system architecture, Modified from [6].

try to deconvolute this reflection response, we present a new algorithm based on mapping and unfolding of relative response between channels measured by drone-borne calibration system. Unlike deconvolution method try to eliminate ground reflection effect for every channel, the gist of unfolding method is to make each channel equivalently go through the same response. With this novel method, not only the interference effect can be alleviated, but also other system responses are calibrated out.

\subsection{Algorithm}

The received waveform in individual channel can be express as:

$$
W_{i}(f, \theta, \phi)=S(f) * P_{i}(f, \vec{r}) * R_{i}(f, \theta, \phi) * X_{i}(f, \theta, \phi)+N_{i}(f),
$$


where $i$ is the label of channel, $S$ represents event source signal, $P$ represents phase change during signal propagate from event source to receiver antenna phase center, $R$ denotes response of ground reflection, $X$ is the system response of individual channel, including antenna, filters and low noise amplifier (LNA), and $N$ is the noise term. $f$ means frequency, $\theta$ and $\phi$ are elevation angle and azimuth angle respectively, $\vec{r}$ is event source coordinate. Phase shift term $P$ between channels contains the information of time-delay-of-arrival (TDOA) which is a prerequisite for event direction reconstruction. But $P$ is entangled with ground reflection term $R$ and system response term $X$.

The relative response between channels is defined as:

$$
\alpha_{i j}(f, \theta, \phi)=\frac{R_{i}(f, \theta, \phi) * X_{i}(f, \theta, \phi)}{R_{j}(f, \theta, \phi) * X_{j}(f, \theta, \phi)} .
$$

$\alpha_{i j}(f, \theta, \phi)$ can be derived with the help of drone-borne calibration pulser system which consists of a solid-state impulse generator, a broad-band transmitter antenna, a differential GPS module that provides an accuracy of centimeter level. This system can be used to scan all angles in our Field of View (FoV) and each cal-pulser event source coordinate can be inferred from its logger. For a cal-pulser event coming from $(\theta, \phi)$ direction, we can define:

$\alpha_{i j}^{m}(f, \theta, \phi)=\frac{W_{i}^{m}(f, \theta, \phi)}{W_{j}^{m}(f, \theta, \phi)} * \frac{P_{j}^{m}(f, \theta, \phi)}{P_{i}^{m}(f, \theta, \phi)} \approx \frac{R_{i}^{m}(f, \theta, \phi) * X_{i}^{m}(f, \theta, \phi)}{R_{j}^{m}(f, \theta, \phi) * X_{j}^{m}(f, \theta, \phi)}+O\left(N_{i}^{m}(f)\right)+O\left(N_{j}^{m}(f)\right)$,

where $m$ is the label of cal-pulser event, and the relative propagation phase shift term $\frac{P_{j}^{m}(f, \theta, \phi)}{P_{i}^{m}(f, \theta, \phi)}$ can be easily calculated by knowing cal-pulser event coordinate. By averaging $\alpha_{i j}^{m}$, the noise term can be further suppressed, thus the relative ground reflection response is derived as:

$$
\alpha_{i j}(f, \theta, \phi) \approx \sum_{m} \alpha_{i j}^{m}(f, \theta, \phi)
$$

For events that need to be reconstructed, unfolding $\alpha_{i j}(f, \theta, \phi)$ on $W_{i}$, the received waveform in channel $j$, we will have:

$$
W_{j}^{\prime}(f, \theta, \phi)=\alpha_{i j} * W_{j}(f, \theta, \phi)=* S(f) * P_{j}(f, \vec{r}) * R_{i}(f, \theta, \phi) * X_{j}(f, \theta, \phi)+N_{j}(f)
$$

The common systematic response of $W_{j}^{\prime}$ and $W_{i}$ can be cancelled out. By cross-correlating, the TDOA information can be extracted out accurately, and thus an interferometric method can be applied here to obtain accurate angular reconstruction result.

Now the problem turns into which relative response $\alpha_{i j}(f, \theta, \phi)$ should be picked up to do the unfolding since the event source direction $(\theta, \phi)$ is not a known argument but a parameter we want to reconstruct. A grid search method is applied here. For each direction in our FoV, an unfolding followed by a cross-correlation is applied for every antenna pair. Since correlation coefficient is a function of hypothetical TDOA that can be calculated for each direction, the average of correlation coefficients over all pairs is thus obtained. If the hypothetical event source direction is correct and the unfolding is done properly, the average correlation coefficient value should close to 1 . The reconstructed direction is thus chosen to be the one that gives the highest average correlation coefficient value. 


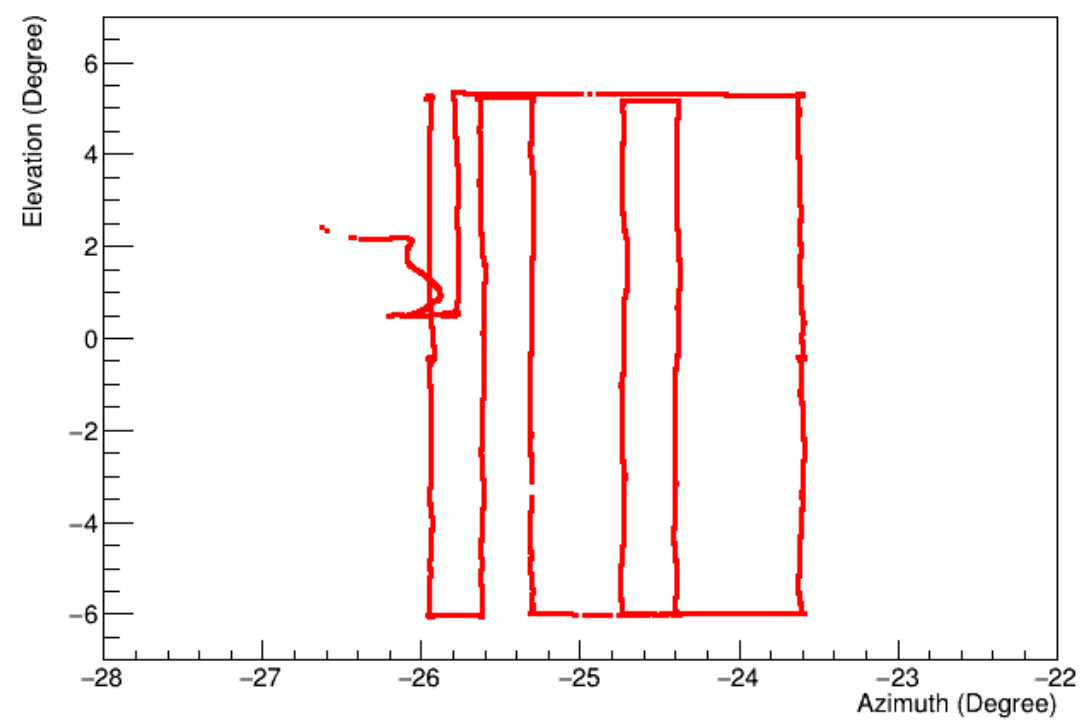

Figure 4: First drone pulser flight path recorded by DGPS. The elevation angle is $0^{\circ}$ in horizontal direction, and azimuth angle is defined as $0^{\circ}$ at geographic east and positive to north, where antennas point to $\sim 27^{\circ}$.

\subsection{Preliminary result}

Two drone flights with $10 \mathrm{~Hz}$ pulsing rate were conducted to verify this algorithm. The first flight made a grid scan at a distance of $\sim 600 \mathrm{~m}$ in the boresight of antenna array as shown in Fig. 4. The scan continues in the vertical direction from $-6^{\circ}$ to $5^{\circ}$ and has $0.5^{\circ}$ step in the horizontal direction from $-27^{\circ}$ to $-23.5^{\circ}$ (antennas point to $-27^{\circ}$ ). There are about 8000 pulser events recorded by the station, those events are then used to obtain relative ground response. Instead of treating as a continuous function of , it is model to be a step function with $0.1^{\circ}$ binning in $\theta$ and $0.5^{\circ}$ binning in $\phi$. The second flight is the verification set which accumulated about 1500 pulser events at a distance of $\sim 800 \mathrm{~m}$ inside the first flight scanning angle range.

The result of reconstruction is shown in Fig. 5 (all 1500 pulser events), angular resolution in elevation and azimuth is $0.67^{\circ}$ and $0.12^{\circ}$ respectively. The angular resolution as a function of event source elevation angle $\theta$ is shown in Fig. 6 . Even for signals coming from $-3^{\circ}$, a sub-degree angular resolution has achieved.

\section{Future works}

In near future, we will scan all angles in TAROGE-4 FoV, and further verify this new angular reconstruction method. Then this method will also be applied into other TAROGE stations in Taiwan and TAROGE-M station in Antarctica.

\section{Acknowledge}

We would like to thank the support from Taiwan Ministry of Science and Technology (MOST). 

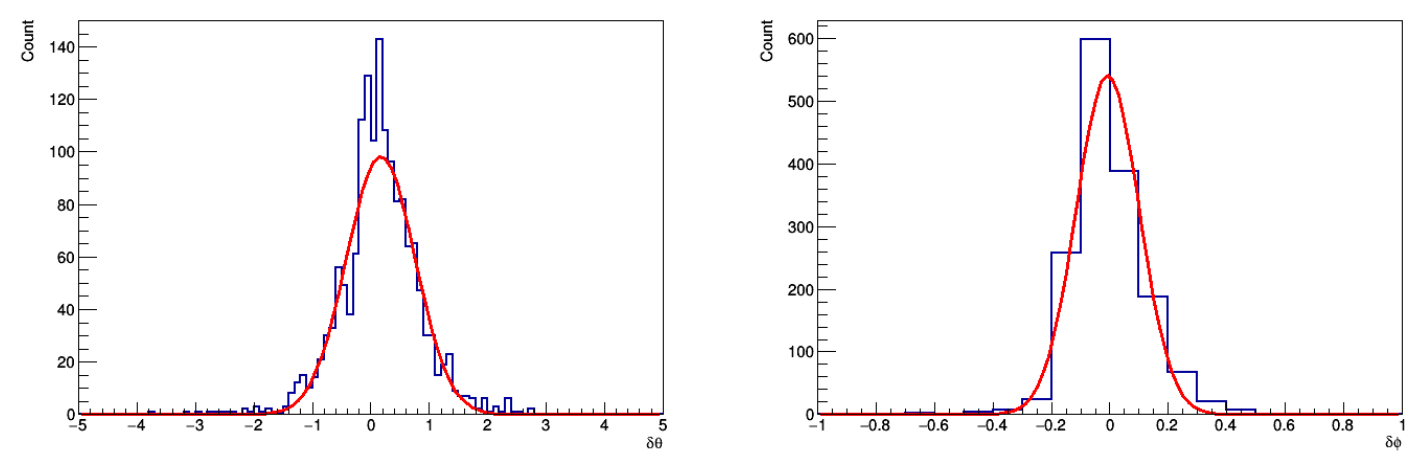

Figure 5: Angular resolution in elevation (Left) and azimuth (Right).
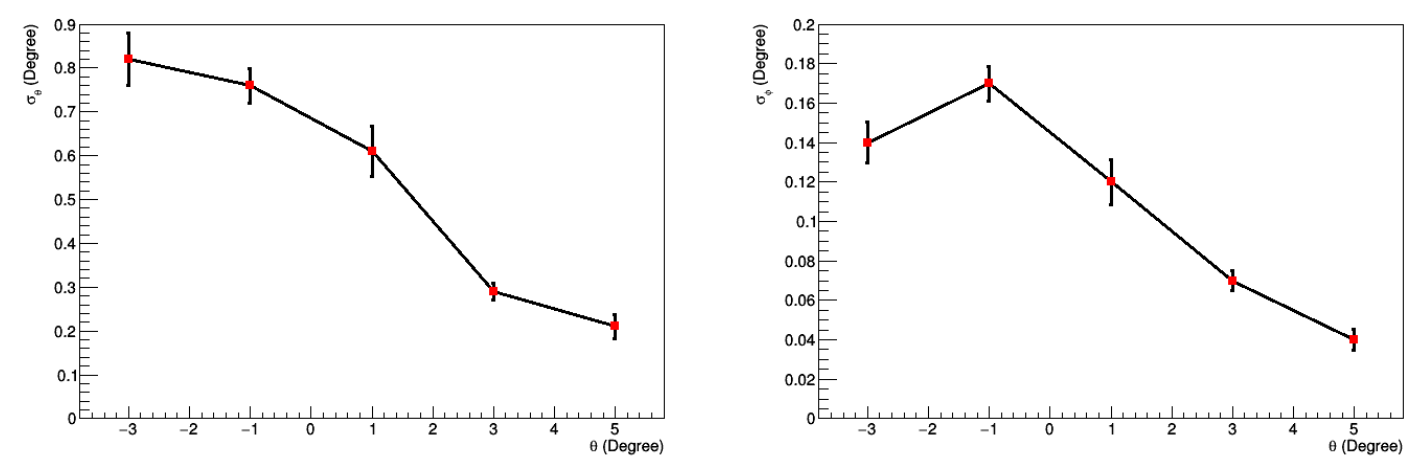

Figure 6: Left: Reconstruction elevation angular resolution versus event source elevation angle. Right: Reconstruction azimuth angular resolution versus event source elevation angle.

\section{References}

[1] F. Schröder, Prog. Part. Nucl. Phys. 93 (2017) 1.

[2] J. Abraham et al., Phys. Rev. Lett. 101 (2008) 061101.

[3] R. U. Abbasi et al. Phys. Rev. Lett. 100 (2008) 101101.

[4] T. Liu et al. PoS(ICRC2017) 234.

[5] C. Kuo et al., PoS(ICRC2021)1429.

[6] J. Nam et al., Int. J. Mod. Phys. D 25 (2016) 1645013.

[7] A. Romero-Wolf et al., Astropart.Phys. 60 (2015) 72. 


\section{Full Authors List: TAROGE Collaboration}

\section{TAROGE Collaboration}

Pisin Chen ${ }^{1,2,3}$, Yaocheng Chen ${ }^{1,2}$, Jian-Jung Huang ${ }^{1,2}$, Ming-Huey Alfred Huang ${ }^{4}$, Chung-Yun Kuo ${ }^{1,2}$, Chung-Hei Leung ${ }^{1,2}$, TsungChe Liu ${ }^{1,5}$, Jiwoo Nam ${ }^{1,2}$, Yu-Shao Jerry Shiao ${ }^{1}$ Min-Zu Wang ${ }^{1,2}$, Shih-Hao Wang ${ }^{1,2}$, Yu-Hsin Wang ${ }^{1,2}$

${ }^{1}$ Leung Center for Cosmology and Particle Astrophysics, National Taiwan University, No. 1, Sec. 4, Roosevelt Rd., Taipei 10617, Taiwan, R.O.C.

${ }^{2}$ Department of Physics, National Taiwan University, No. 1, Sec. 4, Roosevelt Rd., Taipei 10617, Taiwan, R.O.C.

${ }^{3}$ Kavli Institute for Particle Astrophysics and Cosmology, SLAC National Accelerator Laboratory, Stanford University, Stanford, CA 94305, U.S.A.

${ }^{4}$ Department of Energy Engineering, National United University, Miaoli, Taiwan, R.O.C.

${ }^{5}$ Department of Electrophysics, National Yang Ming Chiao Tung University, Hsinchu 300, Taiwan, R.O.C.

${ }^{6}$ National Nano Device Laboratories, Hsinchu 300, Taiwan, R.O.C. 